\title{
亚表面引发原子转移自由基聚合构筑温度响应型纳米纤维油水分离膜
}

\author{
李乐乐 $a, b$ \\ 向阳阳 $a, b$ \\ 刘欢 $a, b$ \\ 麻拴红 $a$ 李斌\#, $a$ \\ 马正峰 $a$ 魏强兵 $b \quad$ 于波 $*, a$ 周峰 $*, a$ \\ ( ${ }^{a}$ 中国科学院兰州化学物理研究所 固体润滑国家重点实验室 兰州 730000) \\ ( ${ }^{b}$ 西北师范大学 化学化工学院 生态功能高分子材料教育部重点实验室 甘肃省高分子材料重点实验室 兰州 730070)
}

\begin{abstract}
摘要 亚表面引发聚合是一种用于制备共价嵌入型聚合物刷的新型改性策略. 该方法在发展高稳定性聚合物刷功能化 表界面材料方面具有显著的优势. 本工作利用亚表面引发原子转移自由基聚合(SSI-ATRP)对静电纺丝聚丙烯腈(PAN) 基纳米纤维膜进行亚表面改性，通过接枝聚 $N$-异丙基丙烯酰胺(PNIPAM)制备了温度响应型纳米纤维油水分离膜 (PAN-sg-PNIPAM). 当温度低于低临界溶解温度(LCST)时, PNIPAM 链与水分子之间的强氢键作用使得聚合物链完全 伸展, 分离膜表面亲水且对油滴具有非常低的粘附力，对油水乳液具有非常高的分离效率(达 $98.7 \%$ ); 当温度高于 LCST 时, PNIPAM 链失水收缩, 膜表面变得更加疏水且对油滴的粘附力显著增加, 其油水乳液分离效率显著降低, 仅 为 $9.1 \%$. 此外, 由于共价嵌入聚合物刷的高稳定性, 该分离膜在 $4 \mathrm{kPa}$ 压力下, $20{ }^{\circ} \mathrm{C}$ 和 $45{ }^{\circ} \mathrm{C}$ 之间可逆切换 10 个循环 后，仍能保持非常稳定的渗透通量. 本研究为发展高稳定性的智能型油水分离膜提供了一种新方法. 关键词 亚表面引发聚合; 原子转移自由基聚合; 纳米纤维膜; 温度响应聚合物刷; 可控油水分离
\end{abstract}

\section{Temperature-Responsive Nanofibrous Membranes Fabricated by Subsurface-Initiated Atom Transfer Radical Polymerization for Controllable Oil/Water Separation}
Lele $\mathrm{Li}^{a, b}$
Yangyang Xiang ${ }^{a, b}$
Huan $\mathrm{Liu}^{a, b}$
Shuanhong $\mathrm{Ma}^{a}$
$\operatorname{Bin} \mathrm{Li}^{\#, a}$
Zhengfeng $\mathrm{Ma}^{a}$
Qiangbing $\mathrm{Wei}^{b}$
$\mathrm{Bo} \mathrm{Yu}^{*, a}$
Feng Zhou ${ }^{*, a}$

( ${ }^{a}$ State Key Laboratory of Solid Lubrication, Lanzhou Institute of Chemical Physics, Chinese Academy of Sciences, Lanzhou 730000, China)

( ${ }^{b}$ Key Laboratory of Eco-functional Polymer Materials of the Ministry of Education, Key Laboratory of Polymer Materials of Gansu Province, College of Chemistry and Chemical Engineering, Northwest Normal University, Lanzhou 730070, China)

\begin{abstract}
Subsurface-initiated polymerization is a novel modification strategy for the preparation of covalently embedded polymer brushes. It shows great advantages in the development of polymer brush-functionalized surface with high stability. In this work, the electrospun polyacrylonitrile (PAN) based nanofibrous membrane was modified by subsurface-initiated atom transfer radical polymerization (sSI-ATRP). Covalently embedded poly( $N$-isopropylacrylamide) (PNIPAM) brushes were grafted from nanofibrous membranes to prepare temperature-responsive oil/water separation membrane (PAN-sg-PNIPAM). When the temperature is lower than the lower critical solution temperature (LCST), strong hydrogen bond interaction between PNIPAM chains and water molecules makes polymer chains fully extended. The membranes are hydrophilic and show very low underwater oil adhesion, resulting in a very high separation efficiency for oil-water emulsions (up to $98.7 \%$ ). While the temperature is higher than LCST, PNIPAM chains dehydrate and collapse, the membranes become more hydrophobic and the underwater oil adhesion increases significantly. Thus, the separation efficiency dramatically decreases to as low as $9.1 \%$. In addition, due to the high stability and durability of covalently embedded polymer brushes, the membrane can maintain a very stable permeation flux after reversible switch between $20{ }^{\circ} \mathrm{C}$ and $45{ }^{\circ} \mathrm{C}$ for 10 cycles under a pressure of $4 \mathrm{kPa}$. This study provides a novel method for the development of highly stable, durable and smart oi/water separation membranes.
\end{abstract}

Keywords subsurface initiated polymerization; atom transfer radical polymerization; nanofibrous membranes; temperature-responsive polymer brush; controllable oil/water separation

*E-mail: yubo@licp.cas.cn; zhouf@licp.cas.cn; Tel.: 0931-4968466

\# 李斌, 目前地址: Physik Department, Technische Universität München. James-Franck-Straße 1, D-85748 Garching.

Received September 27, 2020; published January 2, 2021.

Project supported by the National Natural Science Foundation of China (Nos. 52065061, 51805514, 51705507), Bureau of International Cooperation, Chinese Academy of Sciences (No. 121B62KYSB2017009) and Key Research Projects of Frontier Science of Chinese Academy of Sciences (QYZDY-SSW-JSC013). 项目受国家自然科学基金(Nos. 52065061, 51805514, 51705507)、中国科学院国际合作局(No. 121B62KYSB2017009)和中国科学院前沿科学重点研究 项目(QYZDY-SSW-JSC013)资助. 


\section{1 引言}

随着经济社会的快速发展, 水污染问题日益严重, 各种工业产生的油污染对生态环境造成了长期的破 坏 ${ }^{[1-5]}$. 高效油水分离材料与技术可有效地净化含油污 水保护环境. 其中膜分离技术由于其设计紧凑、占地面 积小、操作简单、分离效率高等优点而被认为是最佳的 油水分离方法之一, 然而目前膜分离仍然存在因膜污染 导致通量和分离效率下降、循环稳定性差等诸多问题 ${ }^{[6]}$. 特殊润湿性分离膜由于具有优异的防污染性能, 能够突 破对高跨膜压力的依赖, 在油水乳液分离中表现出非常 好的应用前景, 也为新型高性能分离膜的设计开辟了新 的方向 ${ }^{[7-12]}$. 与单一“除水”或“除油”型分离膜相比, “智 能调控” 型分离膜在外界刺激下通过改变膜表面的润湿 性, 从而实现除油和除水分离之间的可逆切换, 达到智 能可控油水分离的目的. 通常智能分离膜可以通过调节

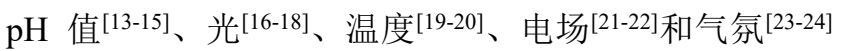
等外部刺激调控表面润湿性, 进而实现智能可控的油水 分离过程.

通过表面改性的方法可以实现表面浸润性的调控. 其中表面接枝聚合物刷是一种广泛使用的表面化学改 性手段 ${ }^{[25]}$, 基于刺激响应性聚合物刷构建的智能表面

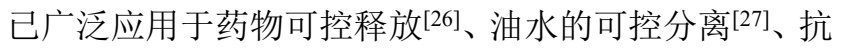
菌-释菌 ${ }^{[28-29]}$ 、界面润滑及摩擦性能调控 ${ }^{[30-32]}$ 等方面. 例 如, 王等 ${ }^{[33]}$ 利用两亲嵌段共聚物(聚 2-乙烯基吡啶- $b$-聚 二甲基硅氧烷, P2VP- $b$-PDMS)对织物表面进行改性, 制 备了水下超亲油/水下超疏油可逆切换表面, 由于接枝 嵌段共聚物中的 P2VP 链段可以在不同 $\mathrm{pH}$ 值下通过质 子化和去质子化改变其润湿性和链构象, 从而实现可切 换的智能油水分离. 除此之外, 温度是最常见、最容易 实现的刺激方法, 被广泛用于调控材料的表面性 质 ${ }^{[34-35]}$. 聚 $N$-异丙基丙烯酰胺(PNIPAM) 是一种典型的 温度响应型聚合物 ${ }^{[36-37]}$, 当温度小于低临界溶解温度 (LCST)时, PNIPAM 链因与水分子之间发生氢键作用而 呈亲水状态, 当温度高于 LCST 时, PNIPAM 链形成分子 间或分子内氢键而使得聚合物链收缩, 呈疏水状态. 江
雷等 ${ }^{[38]}$ 首次采用 PNIPAM 修饰粗粘表面实现温度调控 的超亲水/超疏水之间的可逆切换，此智能表面具有可 逆调控油水分离的性能 ${ }^{[39-41]}$. 近年来, 有关外界刺激调 控的智能油水分离材料的研究报道层出不穷, 但大部分 研究主要集中在 “除水型” 和 “除油型” 之间的可逆切 换. 但是对于 “除油型” 特殊润湿分离膜, 由于水的密 度大于油的密度, 导致膜与油之间产生额外的传递阻 力, 而且当油滴透过分离膜的过程中容易造成膜的污 染, 导致通量严重下降. Vancso 等[42]通过静电纺丝法制 备聚己内酯(PCL)纳米纤维膜, 然后采用 PNIPAM 聚合 物刷进行表面改性, 采用该膜对油水混合物及油水乳液 进行分离, 研究发现 $25{ }^{\circ} \mathrm{C}$ 和 $50{ }^{\circ} \mathrm{C}$ 所对应的分离效率 分别为 $92 \%$ 和 $25 \%$, 实现了对同一种油水乳液(水包油) 的可控分离.

然而传统的聚合物刷存在稳定性不高、耐久性较 差、易破坏脱落等问题，从而造成油水分离效率下降、 使用寿命降低. 近期, 本团队发展了一种新型改性方法, 利用亚表面引发原子转移自由基聚合(sSI-ATRP)可在多 种树脂、水凝胶、静电纺丝高分子纳米纤维膜亚表面制 备共价嵌入型聚合物刷, 实现材料的改性. 与传统聚合 物刷相比, sSI-ATRP 制备的嵌入式聚合物刷以共价键的 方式嵌入到聚合物基底中, 使聚合物刷具有较高的稳定 性，而且嵌入式的亲水性聚合物刷表现出非凡的水合能 力. 研究表明这种亚表面改性的嵌入式聚合物刷在超低 摩擦、抗磨损表面、海洋防污和油水分离方面表现出独 特的优势 $[43-46]$

基于前期工作，如图式 1 所示，本工作利用 SSI-ATRP 对静电纺丝聚丙烯腈(PAN)纳米纤维膜进行亚 表面改性，通过共价接枝 PNIPAM 聚合物刷制备温度响 应型纳米纤维分离膜(PAN-sg-PNIPAM), 利用温度调控 PNIPAM 聚合物链的溶胀与收缩, 成功实现了分离膜对 水下油粘附和油水分离效率的有效调控. 当温度为 $20{ }^{\circ} \mathrm{C}$ 时 $(<\mathrm{LCST})$, PNIPAM 与水分子之间发生强的氢 键作用导致聚合物链完全伸展, 膜表面亲水, 与水相互 作用形成较厚的水化膜且对油滴具有非常低的粘附力,

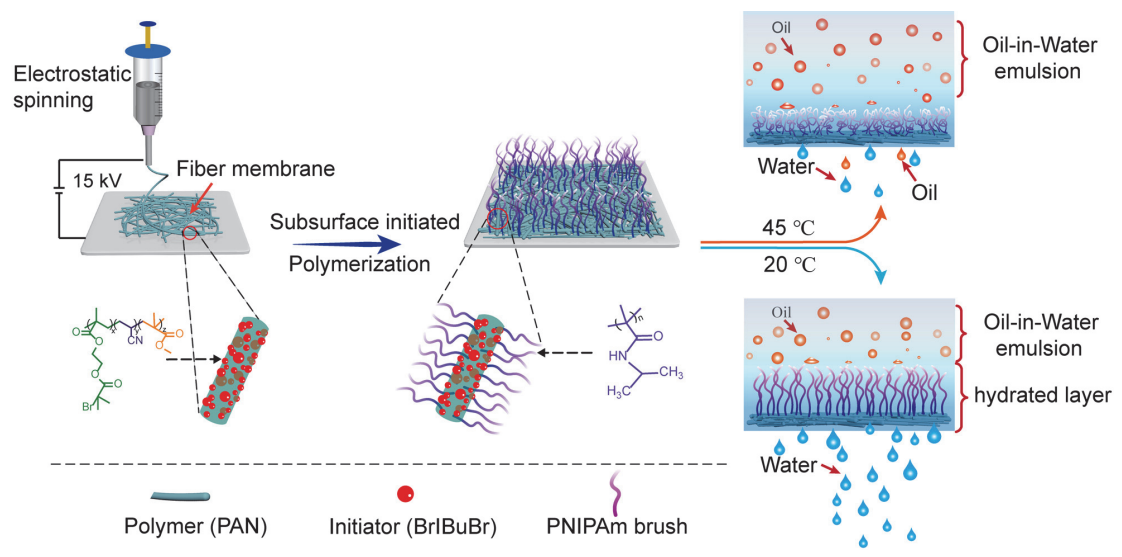

图式 1 温度响应型油水分离膜的制备及分离示意图

Scheme 1 Schematic diagram of preparation and separation process of temperature-responsive oil-water separation membrane 
此时分离膜对油水乳液表现出非常高的分离效率 (98.7\%); 当温度升高至 $45{ }^{\circ} \mathrm{C}$ 时 $(>$ LCST), PNIPAM 聚 合物链失水收缩, 膜表面变得比较疏水, 对油滴的粘附 力显著增加，导致分离效率显著降低(仅为 $9.1 \%$ ).

\section{2 结果与讨论}

\section{1 温度响应型 PAN-sg-PNIPAM 纳米纤维膜的制备 及形貌表征}

首先采用自由基共聚合的方法制备含有 ATRP 引发 剂的 PAN 纺丝液(PAN-Br), 在 PAN-Br 中引入少量聚甲 基丙烯酸甲酯(PMMA) 以降低 PAN 的内聚能, 增加其可 纺性. 其次利用静电纺丝技术制备了 PAN-Br 纳米纤维 膜, 纤维膜中引发剂位点分布在整个纳米纤维的表面和 内部. 然后利用 sSI-ATRP 方法对纳米纤维膜进行亚表 面改性, 制备共价嵌入型的 PNIPAM 聚合物刷. 当纳米 纤维膜在单体的水/异丙醇溶液中接枝聚合时, 纳米纤 维表面溶胀, 促进单体溶液体系扩散到纳米纤维的亚表 面进行接枝聚合, 使聚合物刷嵌入到纳米纤维的亚表 面，从而实现亚表面改性.

接下来对比了纳米纤维膜亚表面改性前后表面形 貌和纤维直径分布的变化, 图 1a 所示为空白 PAN-Br 纤 维膜的扫描电镜(SEM)形貌图, 空白 PAN-Br 纤维膜具 有三维网络结构, 纳米纤维都具有高度均一性和随机取 向的结构, 纤维的平均直径约为 $220 \mathrm{~nm}$, 图 $1 \mathrm{~b}$ 为聚合 2 $\mathrm{h}$ 后 PAN-sg-PNIPAM 纤维膜的 SEM 形貌和纤维平均直 径分布图，由图可以看出，PNIPAM 亚表面改性后的纳 米纤维膜表面形貌发生了明显的变化，纤维表面变得更 加粗粘, 纤维扭曲变形及相邻纤维之间出现粘接, 纳米 纤维直径明显增大，平均直径约增加至 $304 \mathrm{~nm}$ ，这表明 PNIPAM 的成功接枝. 然后采用 $X$ 射线光电子能谱 (XPS)对空白 PAN-Br 和 PAN-sg-PNIPAM 纤维膜进行表 征, 如图 2a 所示, 对于 PAN-Br 膜, 约在 $68.0 \mathrm{eV}$ 处的 $\mathrm{Br} 3 \mathrm{~d}$ 信号为纤维表面及亚表面的 ATRP 引发剂中的溴 元素, PNIPAM 接枝后, Br3d 信号变得非常弱. PAN-Br 纤维膜的 N1s 信号峰位于 $399.5 \mathrm{eV}$ (图 2b), PNIPAM 接 枝改性后，在 $398.5 \mathrm{eV}$ 处出现强 N1s 信号峰，而 399.5 $\mathrm{eV}$ 处的 N1s 信号峰完全消失, 这是因为 PNIPAM 中 $\mathrm{N}$ 元素的结合能低于 PAN 中 $\mathrm{N}$ 元素的结合能，也说明 PAN-Br 纳米纤维表面被 PNIPAM 层完全覆盖. 总之, SEM 形貌的变化、纤维直径的增加和 XPS 表面元素分 析均表明 PAN-sg-PNIPAM 纳米纤维膜的成功制备.

\section{2 温度响应型 PAN-sg-PNIPAM 纳米纤维膜的润湿 性}

纳米纤维分离膜对水和油的润湿性差异是影响其 油水分离效率的重要因素. 因此, 首先通过水接触角测 量(water contact angle, WCA)评价温度响应型纤维膜在 不同温度下的润湿行为. 如图 $3 \mathrm{a}$ 所示, 未改性的 PAN-Br 膜的初始 WCA 为 $126.0^{\circ}$, 经过 $60 \mathrm{~s}$ 后, WCA
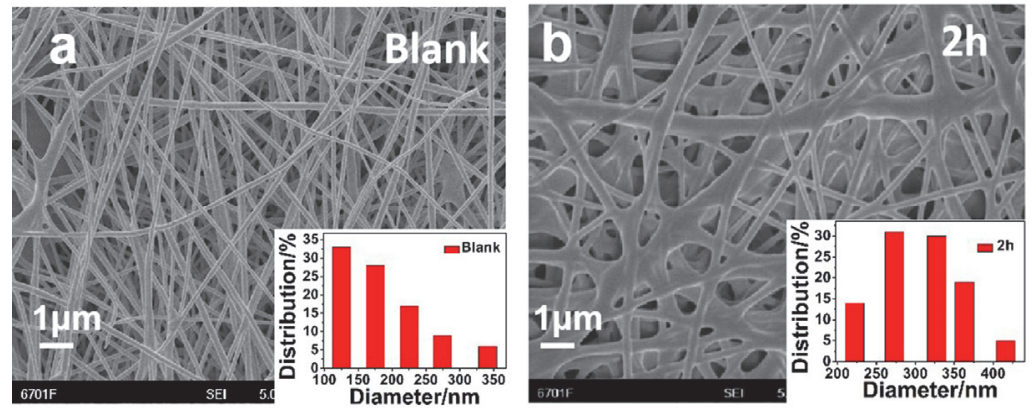

图 1 空白 PAN-Br 膜(a)和 PAN-sg-PNIPAM 膜(b)的 SEM 表面形貌及纳米纤维膜的直径分布

Figure 1 The SEM surface morphology nanofibrous membranes and diameter distribution nanofibers of blank PAN-Br membrane (a) and PAN-sg-PNIPAM membrane (b)
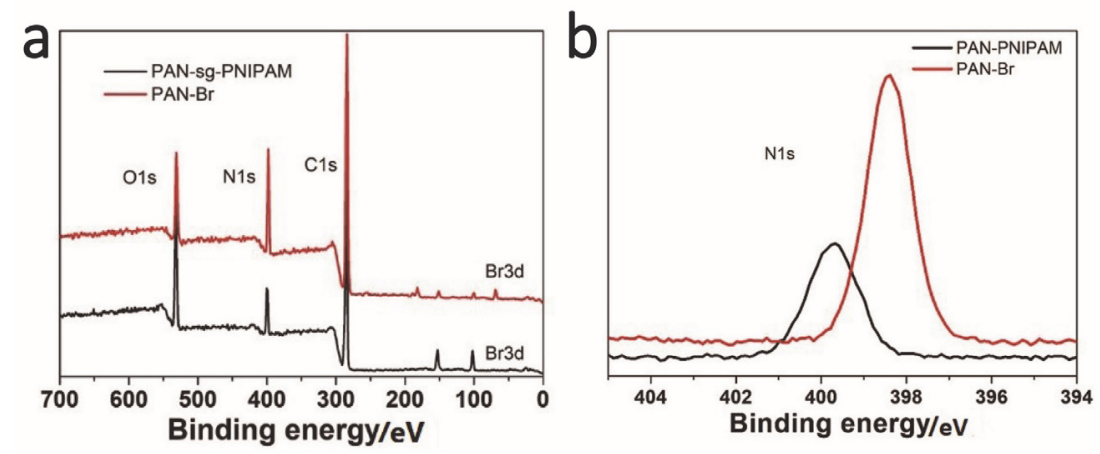

图 2 空白 PAN-Br 和 PAN-sg-PNIPAM 纳米纤维膜的 XPS 表征. (a) XPS 能谱图; (b) N1s 能谱

Figure 2 XPS characterization of blank PAN-Br and PAN-sg-PNIPAM nanofibrous membranes. (a) XPS survey spectra; (b) N1s spectra 
降至 99 ${ }^{\circ}$, 呈疏水状态. 对于 PAN-sg-PNIPAM 膜, 当温 度为 $20{ }^{\circ} \mathrm{C}$ 时, 初始 WCA 为 $76^{\circ}$, 由于 PNIPAM 的亲水 特性, 以及多孔纤维膜的毛细作用的综合影响, 水滴迅 速在纤维膜表面铺展(约 $35 \mathrm{~s}$ ). 当温度升高至 $45{ }^{\circ} \mathrm{C}$ 时, PNIPAM 由于链收缩表现为疏水特征, 其初始 WCA 约 为 $98^{\circ}$, 但水滴仍然很快铺展(约 $30 \mathrm{~s}$ ), 这与文献报道相 一致 ${ }^{[42]} .45{ }^{\circ} \mathrm{C}$ 时水滴在疏水 PAN-sg-PNIPAM 膜表面的 快速铺展表明多孔膜的毛细作用对水滴的铺展和渗透 起主导作用, 这可能是因为 PNIPAM 链收缩, 导致纳米 纤维表面粗糙度增加、膜之间的孔隙相对较大, 使得毛 细作用变得更加显著.

除此之外, 测试了 PAN-sg-PNIPAM 膜在不同温度 时的水下油接触角 (underwater oil contact angle, UWOCA). 将纳米纤维膜贴在玻璃片表面并置于水下, 选用不同种类的油品进行测试. 如图 3b 所示, 在 LCST 以下时, 大豆油(soybean oil)、十六烷(cetane)、异辛烷 (octane)、正已烷(hexane)的 UWOCA 分别为 $150^{\circ} 、 140^{\circ}$ 、 $135^{\circ} 、 134^{\circ}$, 均表现很强的疏油性, 且对大豆油表现出超 疏油特性. 当温度升高至 LCST 以上时, 大豆油、十六 烷、异辛烷和正己烷的 UWOCA 的接触角分别降低至 $127^{\circ} 、 130^{\circ} 、 115^{\circ}$ 和 $107^{\circ}$, 说明随着温度升高, PNIPAM 链收缩并去水化, PAN-sg-PNIPAM 膜的疏油性明显下 降, PAN-sg-PNIPAM 膜在 LCST 以下和以上水下疏油性 的显著变化将会影响其油水分离效率.

\section{3 温度响应型 PAN-sg-PNIPAM 纳米纤维膜的水下 油粘附行为}

为了更好地评价 PAN-sg-PNIPAM 膜在水下的疏油 性能, 我们测试了油滴与膜表面的粘附力, 并用摄像机 原位跟踪了水下油滴与膜表面的粘附过程及其滚动行 为. 如图 4a 所示, 以二氯甲烷为模型液滴, 将探针上的 油滴慢慢下降直到与膜表面相互接触, 然后缓慢分离, 在此过程中同时用力传感器测量油滴与界面之间的粘 附力. 当温度小于 LCST 时, 油滴与膜表面几乎没有粘 附, 油滴从膜表面分离时, 油滴没有变形, 仍然保持球

a

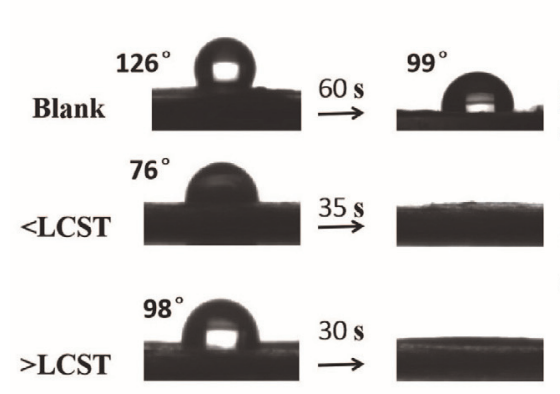

形形状，其粘附力值约为 $7.6 \mu \mathrm{N}$; 相比之下，当温度大 于 LCST 时, 油滴离开纤维膜表面时, 油滴部分粘附在 膜表面, 而且油滴被拉升变形, 测得的粘附力约为 22.7 $\mu \mathrm{N}$ (图 4b), 其值约为低温粘附力的 3 倍. 该结果表明 PAN-sg-PNIPAM 在不同的温度下具有不同的水下油粘 附性能, 在 LCST 以下具有非常低的粘附力, 能够有效 防止油滴的粘附, 有助于提高分离膜的抗油污染能力.

接着研究了不同类型油滴在倾斜 (倾斜角 $=7^{\circ}$ ) PAN-sg-PNIPAM 纳米纤维膜表面的滚动行为, 如图 4c 和 $4 \mathrm{~d}$ 所示, 当温度小于 LCST 时, 无论高粘度的大豆油 还是较低粘度的十六烷均分别在 $30 \mathrm{~ms}$ 和 $18 \mathrm{~ms}$ 内迅速 从膜表面滚落; 当温度大于 LCST 时, 两种油均粘附在 膜表面, 无法从倾斜的膜表面滚落, 这从另一方面也证 明了 PAN-sg-PNIPAM 在温度小于 LCST 时对油品的超 低粘附行为. 这种超低粘附是因为 PNIPAM 聚合物刷在 低温时与水分子之间的强氢键作用所形成的水化层阻 止了油滴的附着. 当温度升高时 PNIPAM 聚合物刷层由 亲水性向亲油性(疏水性)转变, 导致油滴的粘附力大大 增加.

\section{4 温度响应型 PAN-sg-PNIPAM 纳米纤维膜渗透通 量和油水分离的可逆调控}

以十六烷/水为模型油水混合物, 测试了 PAN-sg-PNIPAM 纳米纤维膜在不同外压和温度下的渗 透通量. 如图 $5 \mathrm{a}$ 所示为不同温度时水通量随压力的变 化曲线, 可以看出无论在小于 LCST 还是大于 LCST 的 温度下, 纤维膜的渗透通量都随着压力的升高而增大. 当压力为 $2 \mathrm{kPa}$ 时, $20{ }^{\circ} \mathrm{C}$ 和 $45^{\circ} \mathrm{C}$ 所对应的通量分别为 $1850 \mathrm{~L} \cdot \mathrm{m}^{-2} \cdot \mathrm{h}^{-1}$ 和 $2867 \mathrm{~L} \cdot \mathrm{m}^{-2} \cdot \mathrm{h}^{-1}$, 当压力升高到 $10 \mathrm{kPa}$ 时, 其渗透通量分别增加至 $7500 \mathrm{~L} \cdot \mathrm{m}^{-2} \cdot \mathrm{h}^{-1}$ 和 $8900 \mathrm{~L} \cdot$ $\mathrm{m}^{-2} \cdot \mathrm{h}^{-1}$, 为超高渗透通量. 然而, 在测试压力区间内, $45{ }^{\circ} \mathrm{C}$ 时的通量曲线始终高于 $20{ }^{\circ} \mathrm{C}$ 时的通量变化曲线, 这表明 LCST 以上的通量要明显大于 LCST 以下的渗透 通量. 水通过分离膜的通量主要与膜的孔隙大小、比表

\section{b}

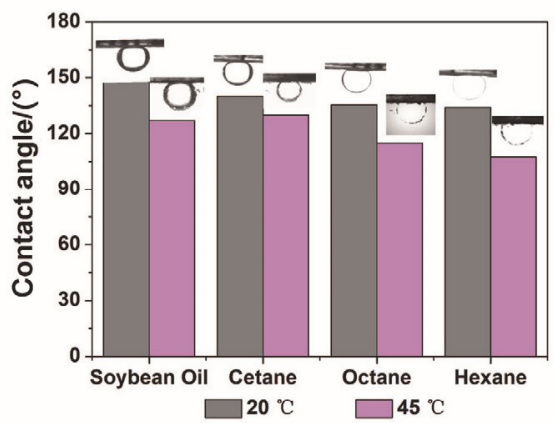

图 3 温度响应型膜的水和水下油的润湿性. (a)空白 PAN 膜和 PAN-sg-PNIPAM 膜在 $20{ }^{\circ} \mathrm{C}(<\mathrm{LCST})$ 和 $45{ }^{\circ} \mathrm{C}(>\mathrm{LCST})$ 的水的接触角; (b) PAN-sg-PNIPAM 膜在 $20{ }^{\circ} \mathrm{C}(<$ LCST $)$ 和 $45{ }^{\circ} \mathrm{C}(>$ LCST $)$ 时水下油的接触角

Figure 3 Water and underwater oil wettability of temperature-responsive membranes. (a) Water contact angles of blank PAN membrane and PAN-sg-PNIPAM membrane at $20{ }^{\circ} \mathrm{C}(<$ LCST $)$ and $45{ }^{\circ} \mathrm{C}(>$ LCST $)$, respectively; (b) underwater oil contact angles of PAN-sg-PNIPAM membrane at $20{ }^{\circ} \mathrm{C}(<$ LCST $)$ and $45{ }^{\circ} \mathrm{C}(>$ LCST $)$ 


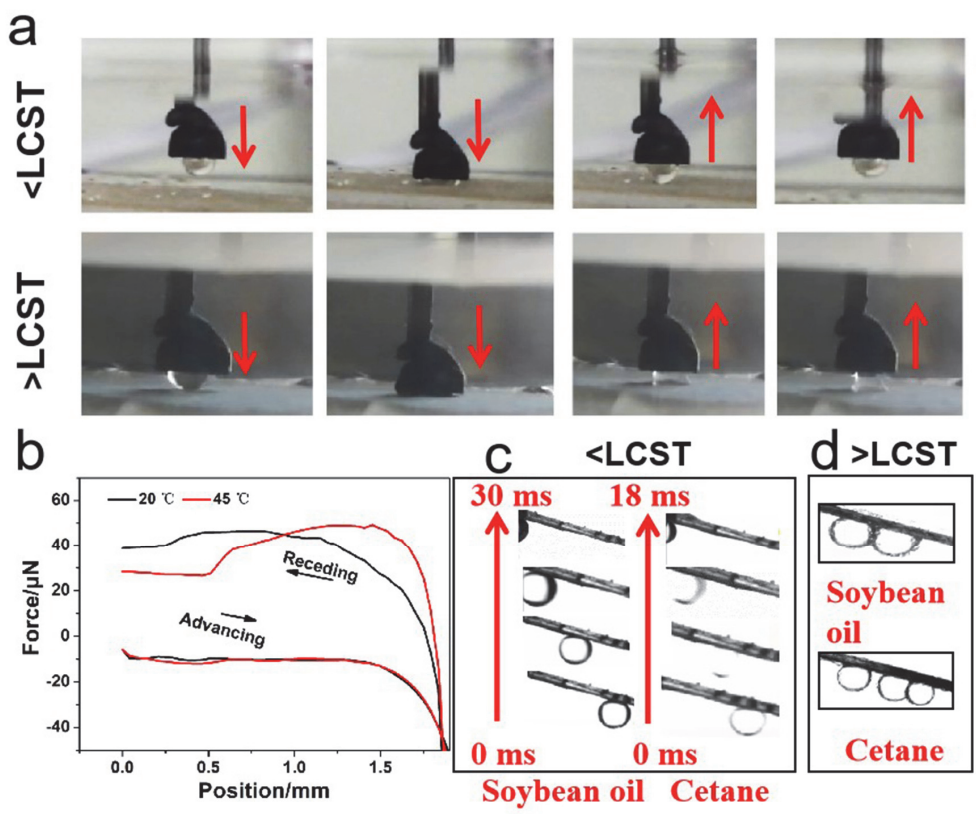

图 4 温度响应 PAN-sg-PNIAPM 膜的水下油粘附性能. (a) $20{ }^{\circ} \mathrm{C}(<\mathrm{LCST})$ 和 $45{ }^{\circ} \mathrm{C}(>$ LCST)时油滴在膜上动态粘附测量的光学图片; (b)动态粘附 测量过程中实时的力-距离曲线; (c) $20{ }^{\circ} \mathrm{C}(<\mathrm{LCST})$ 时不同油滴在水下倾斜膜表面的滚动行为; (d) $45{ }^{\circ} \mathrm{C}(>\mathrm{LCST})$ 时不同油滴在水下倾斜膜表面 的滚动行为

Figure 4 Underwater oil adhesion of temperature-responsive PAN-sg-PNIPAM membrane. (a) Optical snapshots of dynamic adhesion measurements of oil droplet on membranes at $20{ }^{\circ} \mathrm{C}\left(<\right.$ LCST) and $45{ }^{\circ} \mathrm{C}(>$ LCST $)$; (b) real-time recorded force-distance curves during the dynamic adhesion measurements; (c) the underwater rolling behavior of different oil droplets on tilted membrane surface at $20{ }^{\circ} \mathrm{C}(<\mathrm{LCST})$; (d) the underwater rolling behavior of different oil droplets on tilted membrane surface at $45{ }^{\circ} \mathrm{C}(>$ LCST)

面积、亲水性等因素有关. 如图式 2 所示, 当温度高于 LCST 时, PNIPAM 聚合物刷会发生去水化而收缩, PNIPAM 链的收缩会导致纤维膜的孔径增大, 尽管膜表 面变得较为疏水, 但较大的孔径仍然可以使水迅速在膜 表面铺展并渗透到纤维膜内部, 这可能是 $45{ }^{\circ} \mathrm{C}$ 时的水 通量值高于 $20{ }^{\circ} \mathrm{C}$ 时的主要原因. 图 $5 \mathrm{~b}$ 显示了纤维膜在 $4 \mathrm{kPa}$ 压力时, 其在 $20{ }^{\circ} \mathrm{C}$ 和 $45{ }^{\circ} \mathrm{C}$ 时渗透通量和油水分 离的可逆切换, 由图可以看出在 $20{ }^{\circ} \mathrm{C}$ 和 $45{ }^{\circ} \mathrm{C}$ 之间连 续切换 10 个循环后, 通量的变化曲线依然保持稳定, 几 乎没有明显的衰减, 而且可以在高低温之间可逆切换. 对于油水分离性能, $20{ }^{\circ} \mathrm{C}$ 时表现出高的分离效率, 分离 后水中油的含量约为 $25 \mathrm{mg} / \mathrm{L}$, 当温度为 $45{ }^{\circ} \mathrm{C}$ 时, 分离 效率显著降低, 水中油含量上升至大约 $80 \mathrm{mg} / \mathrm{L}$. 而且 在 $20{ }^{\circ} \mathrm{C}$ 和 $45{ }^{\circ} \mathrm{C}$ 之间切换 10 个循环后, 分离效率也可 以实现可逆转变, 并保持稳定, 几乎没有衰减. 10 个循 环后正已烷的水下接触角约为 $116^{\circ}$, 与分离前的 $130^{\circ}$ 相比, 略有下降, 但仍能保持稳定的油水分离调控, 这 充分说明了 PNIPAM 亚表面改性纤维膜的稳定性和耐 久性. 为了进一步证明纤维膜的稳定性, 测试了 PAN-sg-PNIPAM 循环分离后的力学性能, 如图 5c 所示, 未改性的 PAN-Br 膜的拉伸应力小于 $5 \mathrm{MPa}$, PNIPAM 接 枝后, PAN-sg-PNIPAM膜的拉伸应力增加至 $12 \mathrm{MPa}$, 说 明嵌入型聚合物刷的接枝可以显著提高纤维膜的力学 性能. 当经过 10 个可逆分离循环后, PAN-sg-PNIPAM膜 的拉伸应力为大约 $11 \mathrm{MPa}$, 与分离前相比虽然略有下
降, 但仍然能保持比较好的力学性能, 这也进一步说明 PNIPAM 亚表面改性纤维膜的稳定性.

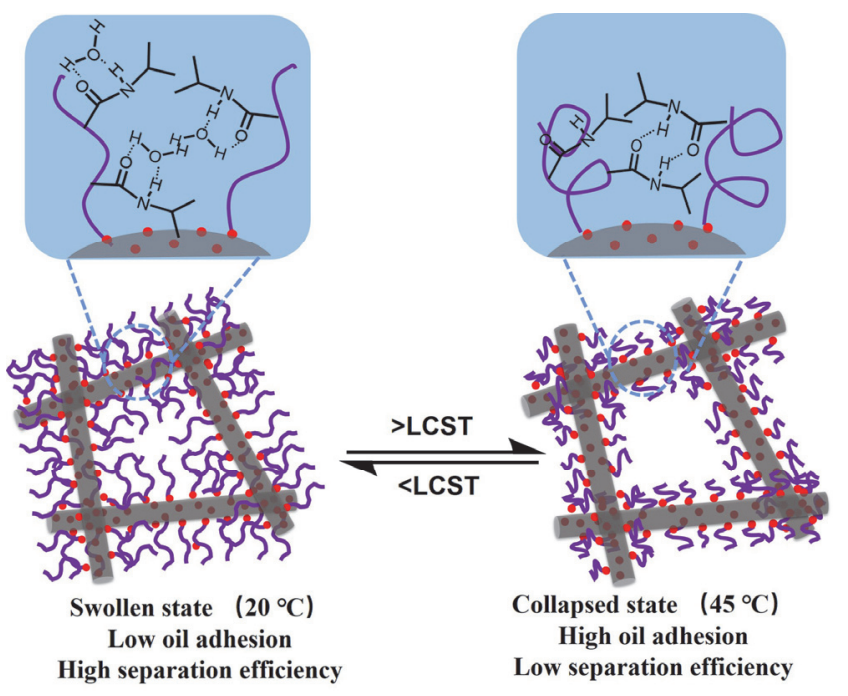

图式 2 温度响应型 PAN-sg-PNIPAM 纤维膜在不同温度下的构象变 化

Scheme 2 The conformational changes of the temperature-responsive PAN-sg-PNIPAM membranes at different temperatures

\section{5 温度响应纤维膜的可调控油水乳液分离}

为了研究 PAN-sg-PNIPAM 纳米纤维膜的可控油水 乳液分离性能, 在 $2 \mathrm{kPa}$ 的压力下进行了油水乳液的分 离实验. 图 $6 \mathrm{a} \sim 6 \mathrm{c}$ 分别为油水乳液在分离前和分离后 

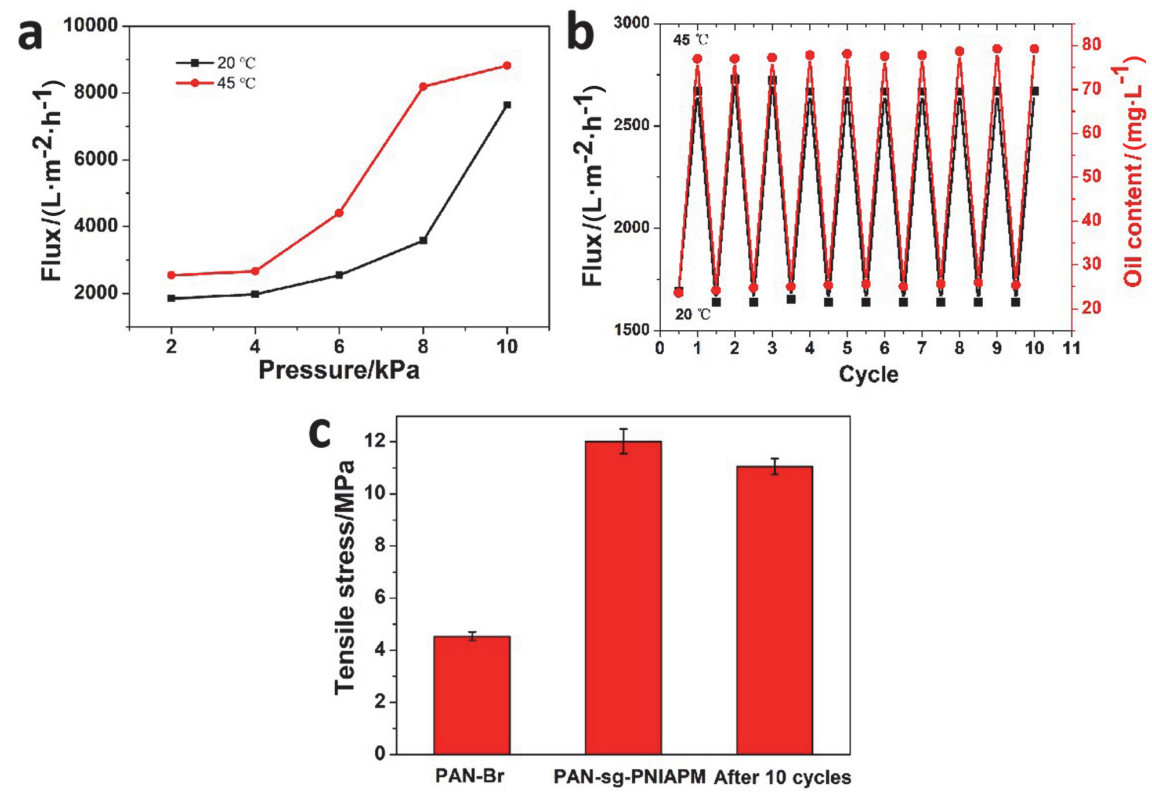

图 5 温度响应 PAN-sg-PNIPAM 膜的通量和力学性能. (a)压力在 $2 \sim 10 \mathrm{kPa}$ 范围内在 $20{ }^{\circ} \mathrm{C}(<\mathrm{LCST})$ 和 $45{ }^{\circ} \mathrm{C}(>\mathrm{LCST})$ 的通量; (b)膜通量在 $4 \mathrm{kPa}$ 时 20 45 ${ }^{\circ} \mathrm{C}$ 之间的切换; (c) PAN-Br, PAN-sg-PNIPAM 和 PAN-sg-PNIPAM 膜经 10 次油水分离后的力学性能

Figure 5 Flux and mechanical properties of temperature-responsive PAN-sg-PNIPAM membrane. (a) Flux at $20{ }^{\circ} \mathrm{C}(<\mathrm{LCST})$ and $45{ }^{\circ} \mathrm{C}(>\mathrm{LCST})$ at the pressure in the range of $2 \sim 10 \mathrm{kPa}$; (b) flux of membrane at $4 \mathrm{kPa}$ switched between $20{ }^{\circ} \mathrm{C}$ and $45{ }^{\circ} \mathrm{C}$; (c) the mechanical properties of PAN-Br, PAN-sg-PNIPAM and PAN-sg-PNIPAM membrane after 10 cycles of oil/water separation tests

的光学显微镜照片, 未经分离的油水乳液在显微镜下可 以观察到大量微米和亚微米级的油滴分布, 动态光散射 (DLS)粒径分析表明乳液由微米和纳米液滴共同组成, 其尺寸从约 $100 \mathrm{~nm}$ 到几微米, 具有较宽的分布(图 $6 \mathrm{a}_{1}$ ). 图 $6 \mathrm{~b}$ 和 $6 \mathrm{c}$ 为油水乳液在不同温度下分离后滤液的光学 显微镜照片, 由图可以看出当温度小于 LCST 时, 分离 后的水相滤液中没有观察到油滴的存在, 而当温度大于 LCST 时, 分离后的滤液中仍然有大量油滴存在. 进一 步对分离后的滤液进行 DLS 粒径分析, 结果表明, 温度 小于 LCST 时, 除尺寸非常小的纳米油滴 $(<200 \mathrm{~nm}$ ) 外, 其余所有油滴均已从水中去除(图 $6 b_{1}$ ), 部分小尺寸纳 米液滴的残留可能是因为分离膜的较宽孔径分布所导 致. 而当温度大于 LCST, 滤液中除了小尺寸纳米液滴 外, 仍然有部分微米级大尺寸油滴残留, 这与显微镜观 察的结果相一致 (图 $6 \mathrm{c}_{1}$ ). 这一结果表明 PAN-sg-PNIPAM 膜在 LCST 以下对表面活性剂稳定的 油水乳状液具有很高的分离效率, 而在 LCST 以上分离 效率很低. 图 $6 \mathrm{~d}$ 为采用苏丹 IV 染料染色的油水乳液, 大于 LCST 时分离之后的滤液仍然呈现出明显的染料颜 色, 而且呈浑浊状态, 而小于 LCST 时分离之后的滤液 完全澄清且无色透明. 由于采用了苏丹 IV 染料对十六 烷进行染色, 所以可以通过紫外可见分光光度法检测染 料的吸收峰, 并采用朗伯比尔定律计算出油水分离效 率. 图 6e 所示为油水乳液在分离前后的紫外可见吸收 光谱, 分离前的乳液在 $360 \mathrm{~nm}$ 和 $519 \mathrm{~nm}$ 处均出现较强 的吸收峰, 在 $20{ }^{\circ} \mathrm{C}$ 时分离后的滤液中染料的吸收峰几 乎完全消失, 这也说明油滴基本被完全除去, 通过计算,
分离效率高达 98.7\%(图 6f). 相比之下, $45{ }^{\circ} \mathrm{C}$ 时分离的 乳液在 $360 \mathrm{~nm}$ 和 $519 \mathrm{~nm}$ 处仍有很强的吸收峰, 这表明 当温度大于 LCST 时对油水乳液分离效率很低, 计算得 到的分离效率仅为 $9.1 \%$ (图 6f), 与 $20{ }^{\circ} \mathrm{C}$ 相比, 分离效 率下降了 $90 \%$ 以上. 这是因为 PNIPAM 聚合物刷在 LCST 以下，高度水化的亲水多孔纤维膜的毛细作用可 以选择性地透过水分子, 其水下超疏油特征和超低油粘 附力可以有效防止油滴对表面的润湿和污染, 因此 PAN-sg-PNIPAM 纳米纤维膜在 $20{ }^{\circ} \mathrm{C}$ 时展现出高的分 离效率. 反之, 当温度上升到 $45{ }^{\circ} \mathrm{C}(>\mathrm{LCST})$ 时, 一方 面 PNIPAM 聚合物刷处于收缩状态, 去水化后的纤维膜 表面疏油性变弱, 而且表现出高粘附力, 这使得油滴很 容易渗透通过纤维膜; 另一方面, LCST 以上渗透通量 的增加也会导致分离效率降低.

\section{3 结论}

本工作采用 sSI-ATRP 对静电纺丝PAN-Br 纳米纤维 膜进行亚表面改性，通过接枝 PNIPAM 制备了温度响应 型纳米纤维油水分离膜(PAN-sg-PNIPAM), 利用温度调 控 PNIPAM 聚合物链的溶胀与收缩, 成功实现了分离膜 对水下油滴粘附力、渗透通量和油水分离效率的可逆调 控. 当温度为 $20{ }^{\circ} \mathrm{C}$ 时 $(<\mathrm{LCST})$, PNIPAM 聚合物链与水 分子之间的强氢键作用导致聚合物链完全伸展, 膜表面 亲水且对油滴具有非常低的粘附力, 该分离膜对油水乳 液表现出非常高的分离效率(达 $98.7 \%$ ); 当温度升高至 $45{ }^{\circ} \mathrm{C}$ 时 $(>$ LCST), PNIPAM 聚合物链失水收缩, 膜表面 

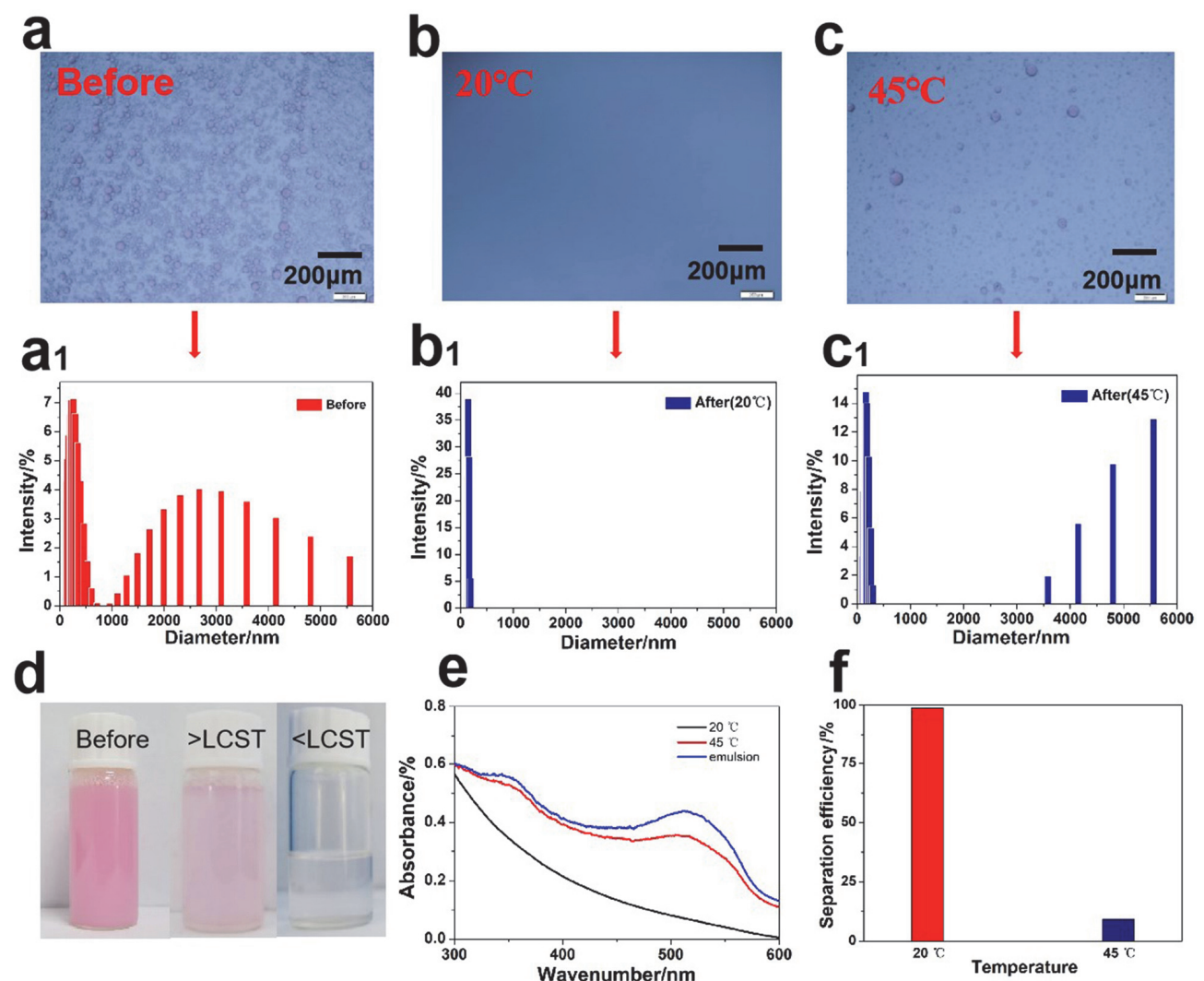

图 6 使用温度响应PAN-sg-PNIPAM 膜在 $20{ }^{\circ} \mathrm{C}\left(<\right.$ LCST)和 $45{ }^{\circ} \mathrm{C}\left(>\right.$ LCST)时乳液的可控油水分离. $(\mathrm{a} \sim \mathrm{c})$ 乳液分离前后的光学显微图像; $\left(\mathrm{a}_{1} \sim \mathrm{c}_{1}\right)$ 油滴粒径分布的动态光散射(DLS)分析图; (d)染色乳液分离前后的光学显微图像; (e)乳液分离前后的紫外吸收光谱; (f)在 $20{ }^{\circ} \mathrm{C}$ 和 $45{ }^{\circ} \mathrm{C}$ 的分离效 率

Figure 6 Controllable separation of emulsion using temperature-responsive PAN-sg-PNIPAM membrane at $20{ }^{\circ} \mathrm{C}(<\mathrm{LCST})$ and $45{ }^{\circ} \mathrm{C}(>\mathrm{LCST})$. (a $\sim$ c) Optical microscopic images of emulsion before and after separation; $\left(\mathrm{a}_{1} \sim \mathrm{c}_{1}\right)$ dynamic light scattering (DLS) analysis of oil droplets size distribution; (d) optical images of dyed emulsion before and after separation; (e) UV spectra of emulsion before and after separation; (f) separation efficiency at $20{ }^{\circ} \mathrm{C}$ and $45{ }^{\circ} \mathrm{C}$

变得更加疏水, 对油滴的粘附力显著增加, 而且分离效 率显著降低 $90 \%$ 以上(仅为 $9.1 \%$ ). 纳米纤维内部共价嵌 入型的 PNIPAM 聚合物链, 使得该温度响应分离膜具有 高的稳定性和耐久性, 该分离膜在 $4 \mathrm{kPa}$ 压力下, $20{ }^{\circ} \mathrm{C}$ 和 $45{ }^{\circ} \mathrm{C}$ 之间可逆切换 10 个循环后, 仍能保持非常稳定 的渗透通量. 本工作所报道的基于亚表面引发聚合的方 法制备温度响应型纳米纤维膜为发展高稳定性、可控粘 附和智能型油水分离膜提供了一种新思路.

\section{4 实验部分}

\section{1 实验试剂与测试仪器}

实验试剂: 甲基丙烯酸甲酯(MMA, 99.8\%)、2-溴异 丁酰澳( $98 \%$ )、甲基丙烯酸着乙酯(HEMA, $97 \%$ )、 $N$-异 丙基丙烯酰胺(NIPAM，98\%)均购于北京百灵威科技有 限公司. 丙烯腈(AN)、吐温 80、2,2'-联吡啶(bipy)、澳 化亚铜、偶氮二异丁腈(AIBN)、十六烷均购自国药集团 化学试剂有限公司. 丙烯腈(AN)在使用前经过蒸馏纯 化, 收集 76 78 ${ }^{\circ} \mathrm{C}$ 的馏分; AIBN 使用前采用无水甲醇 进行重结晶处理, 并避光保存; 澳化亚铜 $(\mathrm{CuBr})$ 在醋酸
溶液中回流过夜, 过滤并用乙醇洗涤、干燥备用. 含有 ATRP 引发剂的单体(BrMA)合成参照文献 ${ }^{[45]}$, 实验中所 用其它试剂及溶剂未经纯化，直接使用.

测试仪器: 采用 FSM-5600lv 型扫描电子显微镜 (SEM, Japan)进行形貌表征; 采用 Thermo ESCALAB 250xi 型 X 射线光电子能谱仪(XPS, 美国)进行薄膜组成 的定性分析, 结合能用 C1s ( $248.6 \mathrm{eV})$ 进行校准; 静态水 接触角采用 DSA-100 接触角测量仪(德国)进行测试, 采 用 $5 \mu \mathrm{L}$ 水滴作为探针液体; 采用 Malvern 3600 动态光 散射仪(DLS，英国)进行乳液中油滴尺寸的分析测量; 采用 Thermo GENESYS 紫外可见分光光度计(美国)采 集油水乳液的紫外吸收光谱.

\section{2 含有 ATRP 引发剂(PAN-Br)树脂的制备}

采用自由基共聚合的方法制备 PAN-Br 树脂, 将含 有 AN (22.5 g, $0.42 \mathrm{~mol}, 90 \%) 、 \mathrm{MMA}(0.5 \mathrm{~g}, 0.005 \mathrm{~mol}$, $2 \%$ )和二甲基亚砜(DMSO, $23 \mathrm{~mL}$ )的混合溶液在圆底烧 瓶中搅拌, 通氮气脱气 $30 \mathrm{~min}$, 然后加入 BrMA $(2 \mathrm{~g}$, $0.007 \mathrm{~mol}, 8 \%)$ 和引发剂 AIBN (1\%). 将溶液加热至 $60{ }^{\circ} \mathrm{C}$, 在氮气气氛保护下反应 $10 \mathrm{~h}$, 得到纺丝原液. 


\section{3 静电纺丝法制备 PAN-Br 纳米纤维膜}

静电纺丝装置由高压电源 $(0 \sim 50 \mathrm{DC} \mathrm{kV})$ 、微针洜 $(V=10 \mathrm{~mL})$ 、金属针头 $($ 直径 $=0.90 \mathrm{~mm})$ 和铝箔接收板组 成. 该装置将作用于正极的喷丝板连接到高压电源上产 生电场, 将接收板连接到地线上. 在静电纺丝过程中, 先用注射器吸取得到原料液体, 然后调节注射泵的注射 速率使得被纺出的丝在接收板上接收, 待溶剂 DMSO 蒸发后, 纳米纤维形式的固化流被收集到接收板上. 本 实验外加电压为 $15 \mathrm{kV}$, 恒滴注射速率为 $0.7 \mathrm{~mL} / \mathrm{h}$, 接收 距离为 $20 \mathrm{~cm}$.

\section{4 亚表面引发原子转移自由基聚合(sSI-ATRP)}

在 $20 \mathrm{~mL}$ 具支试管中, 将单体溶解于水和异丙醇的 混合溶剂中, 通氩气 $30 \mathrm{~min}$ 以排除溶剂中的空气, 并使 单体完全溶解, 然后向溶液中依次加入 $2,2^{\prime}$-联吡啶和 $\mathrm{CuBr}$, 氩气保护下继续摚拌 $15 \mathrm{~min}$, 直到形成均一的红 棕色溶液后, 将引发剂修饰的纳米纤维膜置于该试管 中, 开始聚合反应. 反应一定时间后, 取出纤维膜, 用 大量蒸馏水和乙醇冲洗, 然后用氮气流吹干. 聚合单体 溶液的组成如下: NIPAM $4 \mathrm{~g}$ 、 bipy $60 \mathrm{mg} 、 \mathrm{CuBr} 40 \mathrm{mg}$ 、 $7 \mathrm{~mL} \mathrm{H}_{2} \mathrm{O}$ /异丙醇 $(V / V=3: 1)$.

\section{5 油水混合物及油水乳液的制备}

油/水混合物的制备是直接将水和十六烷混合, 体 积比为 $1: 1$. 表面活性剂稳定油水乳液的制备是在油 相中先加入 $0.1 \mathrm{~g} / \mathrm{L}$ 的吐温 80 , 同时油相用苏丹 IV 染料 染色, 以水与油体积比为 $100: 1$, 然后采用超声波分散 $1 \mathrm{~h}$. 所制备的表面活性剂稳定的乳液在 $24 \mathrm{~h}$ 内具有高 度稳定性。

\section{References}

[1] Stokstad, E. Science 2010, 328, 1618.

[2] Schnoor, J. L. Environ. Sci. Technol. 2010, 44, 4833.

[3] Wang, B.; Liang, W.; Guo, Z.; Liu, W. Chem. Soc. Rev. 2015, 44, 336.

[4] Shannon, M. A.; Bohn, P. W.; Elimelech, M.; Georgiadis, J. G.; Marinas, B. J.; Mayes, A. M. Nature 2008, 452, 301.

[5] Pezeshki, S. R.; Hester, M. W.; Lin, Q.; Nyman, J. A. Environ. Pollut. 1999, 108, 129.

[6] Belkacem, M.; Matamoros, H.; Cabassud, C.; Aurelle, Y.; Cotteret, J. J. Membr. Sci.1995, 106, 195.

[7] Ma, Q.; Cheng, H.; Fane, A. G.; Wang, R.; Zhang, H. Small 2016, $12,2186$.

[8] Zhu, Y.; Wang, D.; Jiang, L.; Jin, J. NPG Asia Mater. 2014, 6, e101.

[9] Li, W. T.; Yong, J. L.; Yang, Q.; Chen, F.; Fang, Y.; Hou, X. Acta Phys.-Chim. Sinica 2018, 34, 456 (in Chinese). (李文涛, 雍佳乐, 杨青, 陈烽, 方瑶, 侯洵, 物理化学学报, 2018, 34, 456.)

[10] Wu, F.; Pickett, K.; Panchal, A.; Liu, M.; Lvov, Y. ACS Appl. Mater. Interfaces 2019, 11, 25445.

[11] Liang, B.; Zhang, G. Y.; Zhong, Z. X.; Tomoya, S.; Atsushi, H.; Su, Z. H. Chem. Eng. J. 2019, 362, 126.

[12] Cao, Y.; Liu, N.; Fu, C.; Li, K.; Tao, L.; Feng, L.; Wei, Y. ACS Appl.
Mater. Interfaces 2014, 6, 2026.

[13] Parnell, A. J.; Martin, S. J.; Dang, C. C.; Geoghegan, M.; Jones, R. A. L.; Crook, C. J.; Howse, J. R.; Ryan, A. J. Polymer 2009, 50 , 1005.

[14] Zhang, L.; Zhang, Z.; Wang, P. NPG Asia Mater. 2012, 4, e8.

[15] Zhou, Y. N.; Li, J. J.; Luo, Z. H. AIChE J. 2016, 62, 1758.

[16] Qu, D. H.; Wang, Q. C.; Zhang, Q. W.; Ma, X.; Tian H. Chem. Rev. 2015, 115, 7543 .

[17] Tian, D.; Zhang, X.; Tian, Y.; Wu, Y.; Wang, X.; Zhai, J.; Jiang, L. J. Mater. Chem. 2012, 22, 19652.

[18] Zhu, H.; Yang, S.; Chen, D.; Li, N.; Xu, Q.; Li, H.; He, J.; Lu, J. Adv. Mater. Interfaces 2016, 3, 1500683.

[19] Xue, B.; Gao, L.; Hou, Y.; Liu, Z.; Jiang, L. Adv. Mater. 2013, 25, 273.

[20] Zhou, Y. N.; Li, J. J.; Luo, Z. H. Ind. Eng. Chem. Res. 2015, 54, 10714.

[21] Kwon, G.; Kota, A. K.; Li, Y.; Sohani, A.; Mabry, J. M.; Tuteja, A. Adv. Mater. 2012, 24, 3666.

[22] Zheng, X.; Guo, Z.; Tian, D.; Zhang, X.; Jiang, L. Adv. Mater. Interfaces 2016, 3, 1600461.

[23] Kota, A. K.; Kwon, G.; Choi, W.; Mabry, J. M.; Tuteja, A. Nat. Commun. 2012, 3, 1025.

[24] Xu, Z.; Zhao, Y.; Wang, H.; Wang, X.; Lin, T. Angew. Chem., Int. Ed. 2015, 54, 4527.

[25] Li, B.; Yu, B.; Zhou, F. Acta Polym. Sinica 2016, 10, 1312 (in Chinese). (李斌, 于波, 周峰, 高分子学报, 2016, 10, 1312.)

[26] Li, B.; Yu, B.; Ye, Q.; Zhou, F. Prog. Chem. 2015, 27, 146 (in Chinese). (李斌, 于波, 叶谦, 周峰, 化学进展, 2015, 27, 146.)

[27] Cao, Y.; Liu, N.; Fu, C.; Li, K.; Tao, L.; Feng, L.; Wei, Y. ACS Appl. Mater. Interfaces 2014, 6, 2026.

[28] Yu, Q.; Cho, J.; Phanindhar, S.; Linnea, K. I.; Gabriel, P. L. ACS Appl. Mater. Interfaces 2013, 5, 9295.

[29] Wei, T.; Zhan, W. J.; Yu, Q.; Chen, H. ACS Appl. Mater. Interfaces. 2017, 9, 25767.

[30] Wei, Q. B.; Cai, M. R.; Zhou, F. Acta Polym. Sinica 2012, 10, 1103 (in Chinese). (魏强兵，蔡美荣，周峰，高分子学报，2012，10, 1103.)

[31] Wu, Y.; Wei, Q. B.; Cai, M. R.; Zhou, F. Adv. Mater. Interfaces 2015, 2,1400392 .

[32] Wei, Q. B.; Cai, M. R.; Zhou, F.; Liu, W. M. Macromolecules 2013 46, 9368 .

[33] Zhang, L. B.; Zhang, Z. H.; Wang, P. NPG Asia Mater. 2012, 4, e8.

[34] Tang, Z.; Akiyama, Y.; Okano, T. J. Polym. Sci.: Polym. Phys. 2014, 52, 917.

[35] Kim, Y. J.; Matsunaga, Y. T. J. Mater. Chem. B 2017, 5, 4307.

[36] Jean, B.; Lee, L. T. J. Phys. Chem. B 2005, 109, 5162.

[37] Chen, J.; Gong, X.; Yang, H.; Yao, Y.; Xu, M.; Chen, Q.; Cheng, R. Macromolecules 2011, 44, 6227.

[38] Sun, T.; Wang, G.; Feng, L.; Liu, B.; Ma, Y.; Jiang, L.; Zhu, D. Angew. Chem. Int. Ed. 2004, 43, 357.

[39] Li, J.; Zhu, L. T.; Luo, Z. H. Chem. Eng. J. 2016, 287, 474.

[40] Yu, Q.; Shivapooja, P.; Johnson, L. M.; Tizazu, G.; Leggett, G. J.; Lopez, G. P. Nanoscale 2013, 5, 3632

[41] Xue, B. L.; Gao, L. C.; Hou, Y. P.; Liu, Z. W.; Jiang, L. Adv. Mater. 2013, 25, 273.

[42] Liu, Y.; Tas, S.; Zhang, K. H.; De Vos, W. M.; Ma, J. H.; Vancso, G. J. Macromolecules 2018, 51, 8435.

[43] Ma, Z. F.; Liu, Q.; Wu, Y.; Li, B.; Yu, B.; Liu, Q. Z.; Zhou, F. Sci. Sinica Chim. 2018, 48, 1611 (in Chinese). (马正峰, 刘强, 吴杨, 李斌, 于波, 刘钦泽, 周峰, 中国科学:化学, 2018, 48, 1611.)

[44] Liu, H.; Ma, Z. F.; Yang, W. F.; Pei, X. W.; Zhou, F. Eur. Polym. J. 2019, 112, 146

[45] Li, L. L.; Xiang, Y. Y.; Yang, W. F.; Liu, Z. L.; Cai, M. R.; Ma, Z. F.; Wei, Q. B.; Pei, X. W.; Yu, B.; Zhou, F. J. Colloid Interface Sci. 2020, 575, 388

[46] Feng, H. Y.; Ma, Z. F.; Zhang, Y. J.; Liu, F. Z.; Ma, S. H.; Zhang, R.; Cai, M. R.; Yu, B.; Zhou, F. Macromol. Mater. Eng. 2020, 305, 2000135 . 